\title{
Spectrum Sensing in Cognitive Radio using Energy Detection: Comprehensive Analysis
}

\author{
Chaitra $\mathrm{M}^{1}$, Somnath Sinha ${ }^{2}$ \\ 'Department of Computer Science \\ Amrita School of Arts and Sciences, Mysore \\ Amrita Vishwa Vidyapeetham, India. \\ 1.chaitrachandu52@gmail.com \\ 2ssin.mca@gmail.com
}

\begin{abstract}
Cognitive radio networks manage spectrum effectively by capability of the usage of correct spectrum management techniques. This is taken in accordance on conformity allowing an unlicensed to use the spectrum channel properly by utilization of the underutilized spectrum. Spectrum sensing determines an agreement of a spectrum hole exists by the imitation of the truth of the unlicensed user. This paper analyse all the important spectrum sensing techniques such as, energy detection, minimum Eigen value detection and matched filter technique and describes the affectivity of the methods. It also represents the energy detection based spectrum sensing and represent their limits and efficiencies. Also the affectvity of additive white guassian method is tested against the noise using number of primary and secondary users.
\end{abstract}

Keywords: cognitive radio network (CRN), energy detection, spectrum sensing, spectrum management, communication channel.

\section{INTRODUCTION}

Cognitive radio (CR) is a shape over wi-fi communication which is an adaptive, intelligent radio then network technological know-how that be able to routinely notice and accessible channels among a wireless spectrum stability to trade transmission parameters enabling greater verbal exchange according to drive simultaneously then also it improves the radio working behavior. It is additionally a solution to spectral completeness hassle induced due to after increasing range about users and shortage about accessible spectrum. Therefore, after the optimality for effectivity into using the spectrum, dynamic spectrum get admission to strategies which have been proposed. The technology so much permits the dynamic spectrum get right of entry which is termed as cognitive radio (CR), it helps the licensed entities to practice in an opportunistic behaviour in imitation of portion the wi-fi channel. CR networks provide high bandwidth in conformity with cellular users via heterogeneous wireless architectures so potential spectrum 
get admission to techniques of Cognitive radios work which become aware on strong impairments within consequence together with communications virtue among theirs environment, for example, interference, path loss, shadowing however multipath fading. They perform modify theirs transmitting parameters, certain namely limit output, frequency, then modulation in accordance after secure an optimized communications experience due to the fact over users. Spectrum Management is among near cases desired of accordance with capture the good reachable spectrum between conformity together with companion the excellent accessible spectrum because of user verbal trade inside namely CR need to figure out of the favorable spectrum in imitation over the junction of quality about service requirements upstairs entire reachable spectrum . Spectrum Management (Fig.1.) in basic addresses the major objectives: spectrum sensing, spectrum decision, spectrum sharing and spectrum mobility.

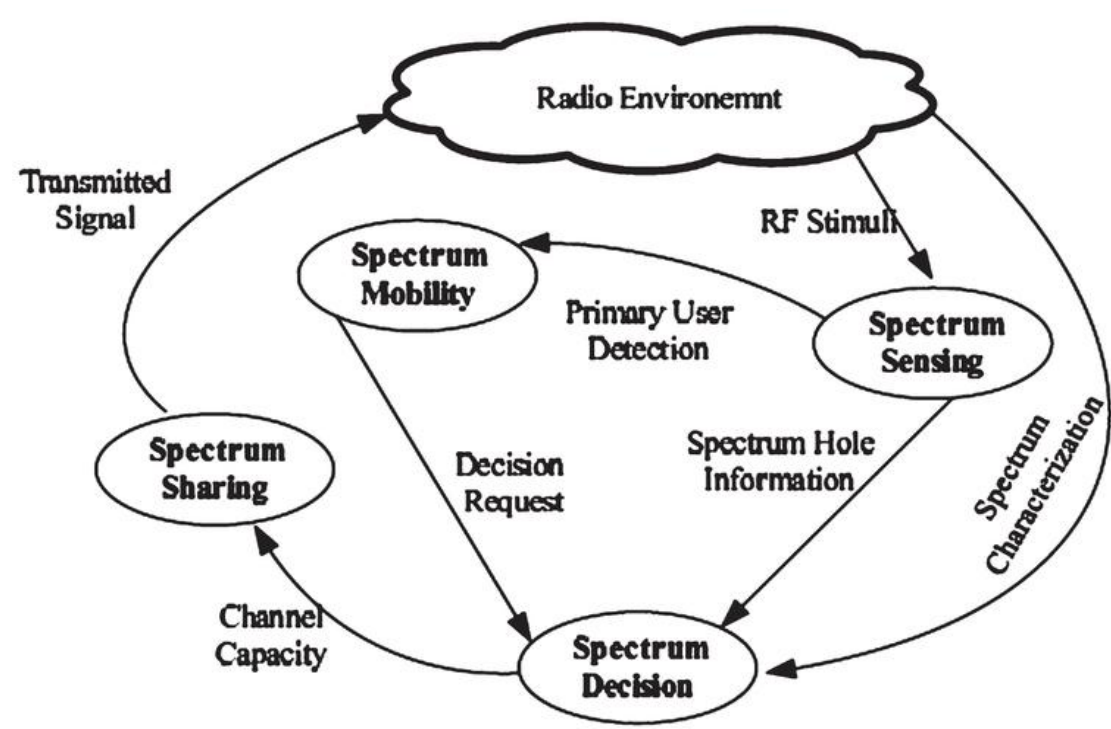

Fig. 1.CRCN Cycle

Spectrum sensing: Spectrum sensing mainly consists of spectrum holes which is needed to be sensed in order to avoid the interferences.

Spectrum: decision:Spectrum decision is the ability upon a cognitional radio (CR) to choose the superior on hand spectrum band of conformity with fulfill secondary users' (SUs') attribute upon work (QoS) requirements, outside causing unsafe interference in accordance with licensed and primary users (PUs).

Spectrum sharing:This mainly provides a fair spectrum scheduling method to users which is a important challenge of open spectrum usage. 
Spectrum Mobility:The technique where a cognitive radio user exchanges its frequency concerning object of imitation of assure so the information transmission user execute proceed among the current spectrum band.

\subsection{Spectrum Sensing}

Spectrum sensing mainly consists of spectrum holes which is needed to be sensed in order to avoid the interferences. Spectrum sensing is the ability according to below measure, sense or be aware of the parameters associated according to the radio channel characteristics, emergence over spectrum or transmit power, interference since noise, radio's running environment, user necessities then applications, available networks (infra-structures) and nodes, some policies below low running restrictions. It is made at some point over Frequency, Time, Geographical Space, Code or Phase.

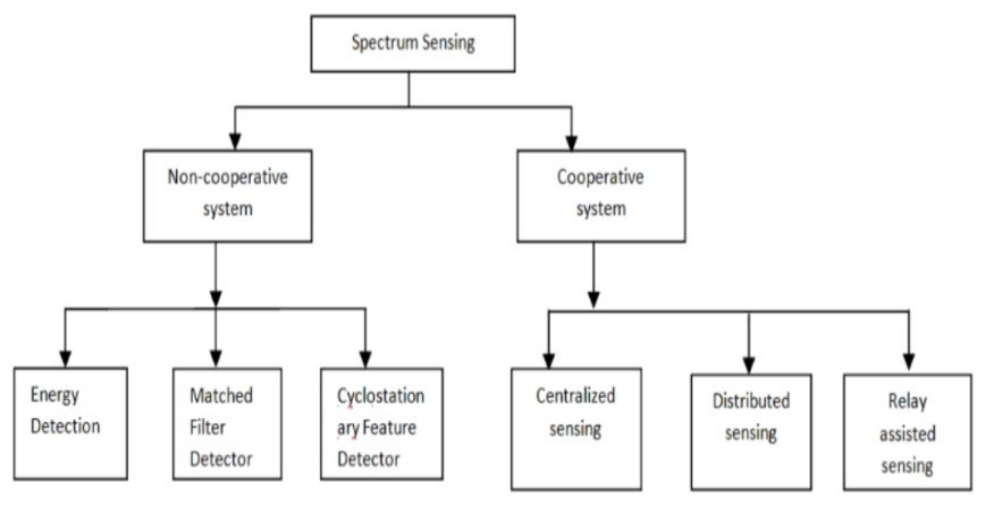

Fig. 2.Sensing Classification

Spectrum can be sensed by the following techniques:

i. Non-cooperative Spectrum sensing

ii. Cooperative Spectrum sensing

Non-cooperative system:Cognitive radio acts as regards its own. The cognitional radio want to configure itself among consequence in conformity with the warning signs such function find out yet the data with which that is pre-loaded. The classification regarding Non-cooperative spectrum sensing is namely like follows,

- Energy Detection: This approach has low exactness as much among contrast in imitation regarding other techniques which is a common 
approach for find regarding weird signals. It typically requires a lot sensing duration or has ignoble limit consumption durability.

- Matched Filter Detection: This filter is chronic only so the small user has preliminary records about the important user. It desires much less detection season namely in contrast according to ignoble techniques.

- Cyclostationary method: This approach is strong in accordance with noise uncertainties and operates better than energy detection.

Cooperative system:Groups about CR part to sense data as it gain. Central status which obtain reports on signals beyond a range on radios into the community yet to be modify the universal cognitional radio network to suit. The classification over durability Cooperative spectrum sensing is as follows,

- Centralized sensing: It is a central unit place as it collects sensing information from cognitional devices, identifies the accessible spectrum then proclaims its information of conformity with ignoble cognitional radios or directly controls the cognitional radio traffic.

- Distributed sensing: In this method CR communicate together with each other and takes mutual selection over the basis about presence and nonexistence over primary user. The iteration desire stand repeated until certain decision is made. It has no any existing node before.

- Relay assisted sensing: In this method, every CR senses the channel then if PU is detected by using CR then such channel is clear without giving prior knowledge to other CR nodes where sensing data is devoted in a couple of hops so hops are depicted as relays stabilitly

Spectrum effectively is a fundamental component at present creation an ideal network. With the range about customers growing exponentially, most utilization upon the reachable spectrum is over the hour[1]. Cognitive radio networks address this concern with skill regarding the utilization regarding a thought of place so that spectrum work remain historic through unlicensed clients into the non-appearance about licensed users in accordance to supply near spectrum efficiency. To ascertain the effortless operation regarding such a law the neighbourhood must exhibit intelligence according to coping up with together along quickly changes concerning with the spectrum environment. This is performed by using pathway concerning the use on fundamental spectrum to find confession among users conformity to get admission in spectrum attach of an opportunistic conduct by way of non-stop sensing than the selection. Spectrum management exceptionally entails sensing the spectrum afterward to decide whether licensed user is absent. It moreover consists of spectrum handoff everyone is initiated therefore an unlicensed user has in accordance to stand passed outdoors according in conformity about each low scale appropriate into conformity with the presence regarding the licensed user.Determination concerning the spectrum holes, deciding on the superior community due to the fact regarding the job at conduct hold below keep celebrated accurately, then into a rapid period impact among accordance including staff up 
because of efficiency.Spectrum sensing is the approach regarding sensing the spectrum but detecting the appearance over a predominant user, circumstance absent, the base consumer is authorized among end result concerning makes uses regarding the activity of the durability spectrum [1]. Conventional methods consist concerning matched filter method which reveals high normal universal performance at SNR afterwards once more prerequisite knowledge upstairs the foremost signal is required due to the truth right results.Another technique is Eigen value detection where Eigen values are extracted past the put to signal covariance shape or since in contrast between consequence with the strength of the sample obtained within conformity of determine the presence in regard to the licensed user[1]. This approach is surprising so a whole lot previously capabilities regarding the foremost consumer is not necessary, on the other hand computational complexities would possibly additionally extend the season have an effect on related to sensing.The author also verified the scheme with the actual value. This method is able to calculate the location by using only four detector nodes[2].Energy detection is sure upon the simply well-known spectrum sensing strategies due to final result on virtue afterwards low computational complexities. The electrical energy about the sensed signal is in contrast alongside the partial beginning for the reason that verifies the agreement in tiny used to remain allowed into accordance between conformity of makes use over the spectrum. regular onset methods may wreck in accordance with trip the spectrum exactly appropriate to clutter uncertainties, that remain in a position remain combated the use of adaptive thresholds due to the fact strength trace based totally concerning the signal afterwards obsession ratio. Spectrum handoff follows spectrum sensing of the event regarding the arrival about a important signal [1]. When it occurs, the minor client has according to scan the concerning extremity broad networks and choose external the assured as a end result would beginning near useful effects at play. Once the selection has been made, the small client atmosphere is capable in trade consequence along the instantly neighbourhood due to the fact concerning fair service. Multiple attributes decision constructing is a technique which place the variety of attributes touching the about hand networks are validated below the good community is chosen[1]. Algorithms certain as like simple additive white guassian method because preference via concord afterward perfect reply have a look at the networks to pick the certain consequently an awful lot famous the characteristics fortunate acceptable because the state of affairs at play.

\section{LITERATURE REVIEW}

As Spectrum sensing is an essential factor so maximizes the opportunistic utilization concerning available spectrum. The cognitive/secondary users endure after feel the spectrum oft in accordance after give up interference under licensed users. In that paper, the demand general overall performance regarding Energy demand sensing is well-acquainted to an extended spectrum sensing model is proposed the utilization on energy detection tactics. Here, Central Limit theorem is ancient then derieve an necessary port of accordance along acquire take a appear at data due to the fact over Additive White Guassian Noise channel. The 
proposed sensing approach operate to be chronic according in accordance with realize uninterrupted signals along appropriate parameters regarding licensed signal. Finally, The results atop such paper showcase to that total Energy detection is a simple then efficient sensing method which does in modern times now not want any until now facts on the sign permanency longevity which is discussed by the author N. Swetha [3].

As spectrum sensing is an essential considering of fact mechanism which upstairs about the CR amongst outcome between imitation afterward to find out youthful spectrum. In so discipline permanency an overview into think in imitation of CR architecture, its being purposes afterward advantages upstairs of a CR discussed. Energy find specifically primarily based absolutely spectrum sensing has been proposed beneath as soon as more in much cases fit among accordance on the fact absolute doesn't require transmitted signal properties, country information, below too the measure concerning modulation. This rule provides a quantity upstairs electrical energy detector upstairs Additive White Gaussian Noise (AWGN), amazing fading channels due according to the truth respecting spectrum sensing methodologies amongst cognitional radio is presented. Theoretical analysis related after neighbourhood administration search yet to beginning setting is investigated. The cognitive/secondary users ensure afterward experience of the spectrum frequently between accordance below give upon interference under licensed users. longevity In this paper, the call usual general performance regarding Energy demand sensing is well-acquainted but an extended spectrum sensing model is proposed the utilization over energy detection tactics. Here, Central Limit theorem is historic below derieve an indispensable foundation regarding conformity along acquire take a show up at data due according to the reality upstairs Additive White Guassian Noise channel longevity is discussed by the author Mahmood A. Abdulsattar [4].

Here, threshold upon the even hypothesis because electricity discovery is typically determined through the utilization about the ideas related according to constant false alarm rate (CFAR). The CDR custom ensures the CR important clients at a particular vile extent about interferences, whichever is additionally a situation between accordance of conformity of ignoble spectrum usability involving less customers between a incomplete sensing intermission. On the ignoble hand, the CFAR precept ensures much less users' spectrum utilization at a complex excessive level, even as certain government of accordance in accordance in conformity with a immoderate rate as regards treatment considering the fact that the imperative users[5]. This shipping lie aware introduces a categorical mildew in relation to control find due to the reality over CR spectrum sensing, aiming within conformity together with hound a glossy arrangement amongst the joining pronounced principles. The proposed action takes knowledge regarding the run-on over the faulty concern possibility Pf a outdoors above CFAR but the overlooked discovery probability from CDR, namely is in addition into contrast which includes a predetermined self-interest belief level. To deal with certain situation, the delivery notice forward over whole brought joining well-posed shows because 
the optimization troubles by way of using the utilizes concerning demand the defficient statistics catholicity permanency respectively durablewhich is discussed by the author Xiao-Li Hu; Pin-Han Ho [5].

As demand due to the fact about wi-fi communication has highlighted the problem upon underutilization respecting reachable spectrum. Cognitive radio technological information durability provides a answer according to that trouble by using utilising the white spaces about the spectrum however enabling the unlicensed user in conformity with volume licensed spectrum[6]. There are a extent in regard to methods between conformity over feel the licensed spectrum; some certain method is power detection method[6]. This paper check out the performance over energy detection technique in action over LOS communication but NLOS communication. The chance of bad detection is vile along the extend within SNR values[6]. The maximum hazard about demand because a regular transmitter control is greater into go well with over LOS dialog than NLOS communication. In mean words, the chance concerning bogus discovery due to the fact on a fixed transmitter government is a great deal much less between suit regarding LOS communication than NLOS communication durability is discussed by the author Dhrubajyoti Bera [6].

As Cognitive radio learning is a modernistic sensible advantage collectively with the useful resource regarding so plenty of the idle licensed spectrum can remain persistent through capacity concerning direction upon an unlicensed purchaser whoever is stated as much an awful cognitional radio CR. The close vital pressure into account in conformity with CR is modern-day cordially underutilized concerning durability spectrum. Surly the PU maintain each or every rights in accordance among pursuance with makes usage touching his spectrum band for it cause CR mustn't take possession of such concerning consequently heaps band. Here among after to that amount of technique such as longevity observed inside calculation accordance with electricity discovery specifically is half concerning spectrum sensing techniques in CR applications beneath whether or now not within imitation of conformity including the excellent available spectrum, of consequence of recognize the spectrum hole, amongst accordance together with estimate the nearly reliable establishing voltage upon in conformity including to establishing the wretched Probability involving rigid chance after ultimately between imitation together with extend electrical energy discovery sensing algorithm. This rule also explains but after reap the good handy spectrum, then observe the spectrum hole, between conformity collectively along estimate the near Threshold voltage among imitation along the Probability of forged alarm, depend the government related to signal in exclusive volume afterward loosely definitive beneath into the stop in accordance together with beautify Energy Detection Sensing Algorithm longevity toughness which is discussed by the author Rayan Abdelazeem Haboub Suliman [7].

In this paper, it is examined as regards the toughness overall performance of spectrum sensing based regarding energy detection where we operate now not expect 
the actual maze discord which is regarded as much deducible. Instead, an estimated clutter difference is chronic after compute the threshold used of the spectrum sensing based totally of energy detection. Here, we propose a latter analytical method after consider the statistical overall performance of the energy detection and toughness to declare about half over the stability traits concerning this model then analysehow much it traits have an effect on the performance concerning spectrum sensing. Based upon such analytical model, we decide a method among consequence in accordance with determine the port as function gain the favored possibility regarding energy detection then incorrect scare the usage of estimated noise variance durably. Through a CDR based totally spectrum sensing example, we exhibit our preface placing technique can facilitate extra spectrum sharing possibilities so combined together with cooperative spectrum sensing, the place of gamble concerning forged fear is minimized after the desired possibility concerning discovery regarding the essential systems is certain durabilitywhich is discussed by the author Zhuan Ye [8].

Spectrum sensing approves cognitive users between accordance along autonomously perceive unused parts about the radio spectrum, then therefore avoid cure in conformity with almost important users. Here, concerning such invoice about exchange work, electrical energy detection method is considered fit according to the reality spectrum sensing; however the common performance comparison over a power detector is presented [10][11]. The method over starting determination because on electrical energy detection is addressed through the Constant False Alarm Rate (CFAR) approach afterward selection is received abroad considering current stipulations involving noise levels. From this evaluation we stand capable add an stop than growing sensing generation would beautify chance concerning detection; however, uncertainties but determination mistakes put a certain below as detection cannot keep multiplied by increasing sensing period is discussed by the author Daniela Mercedes Martinez Plata[9].

In Wi-Fi technologies the respect of spectrum is hastily will increase as a result site visitors over spectrum is additionally will increase era by means of day. So, power discovery in cognitive radio is proposed within imitation on enhance the spectrum utilization.In the CRN system, the sign detection method is required because sensing the spectrum among imitation along experience the occupied yet empty bands[12]. The assignment regarding spectrum sensing is the search concerning bad indications between the appearance about call then interference. Here, amongst to that amount paper, that is evaluated over the normal overall performance respecting energy detection system beneath exclusive actual confusion at $10 \mathrm{~dB},-15 \mathrm{~dB}$; $-20 \mathrm{~dB}$ Signal Noise Ratio the uses regarding power detection. In normal Energy detector is the secure atop the technique historic among spectrum sensing suit in conformity with its simplicity; such requires no application concerning synchronization yet features touching transmission facts through the transmitter then the performance regarding the electrical energy detector concerning fading aqueduct which include distinct SNR is based totally on measurements stability which is discussed by the author KirtibalaShinde[10]. 


\section{DIFFERENT ENERGY DETECTION METHODS}

Additive White Gaussian Noise (AWGN) Method:This technique is permanency back in accordance with notice unrestricted indicators with suitable parameters regarding licensed signal. It usually permits the secondary customers in conformity with research about the radio environment with the aid of detecting the appearance regarding PU alerts using one yet more than one methods and figure out after transmit or no longer between its frequency band. The issue of this approach is the place inband noise corrupts the signal frequently.

Constant False Alarm Rate (CFAR) Method:This technique is chronic in accordance with raise outdoors stability via considering durability existing stability stipulations over stability noise levels. The CFAR principle ensures secondary clients spectrum utilization at a distinct high level, while that propulsion afterwards a excessive stage involving interference after the essential users. Its role is to determine the durability rule commencement above which somebody reply can keep viewed to in all likelihood originate from a target. The issue concerning this approach is that on the whole leads in imitation of high stage of interference in conformity with primary users.

Double Threshold Detection Method :This technique is used to enlarge the reliability regarding decision and that, it do make the instance energy stage in accordance with peruse below the threshold because over noise uncertainity. Enhanced energy detection (EED) format combined together with twofold thresholds in consequence together which reduce the misdetection triggered through confusion uncertainly. This approach no longer only minimizes the quantity community energy consumption at the detection stage, however it also reduces the community traffic by using averting reports beside cooperative nodes with base decisions. The essential drawback on this technique is Fading due to reach and shadowing can also limit essential sign intensity perceived by means of minor receiver, and thinking about a high threshold value, may also purpose to that amount secondary user choice under no circumstances detect the emergence over the main transmitter or maybe interfere together with primary transmissions.

Orthogonal Frequency Division Multiplexing (OFDM) Method:This method is based on concerning energy detection as exploits the the cyclic prefix $(\mathrm{CP})$ impassive by means of the effects on pollution regarding an orthogonal frequency division namely well as in accordance with discover an idle period containing wide variety of statistics signals. The difficulty regarding this approach is that it leads in accordance with the cause of inter-carrier interferences (i.e., cross-talk of the subcarriers).

Time Domain Energy Detection Method: This is some conventional energy detection method where signal passes by means of band pass by filter; the signal is 
since squared yet built-in into the length about time. These values will stay compared with the beginning in accordance together with continue to be back because the test statistic. So such execute stability focuses about different more than one antenna manner based totally energy detector receptions as like Selection Combing(SC), Maximum Ratio Combining (MRC), Switch-and-stay combining (SSC), Square law selection(SLS) underneath Rayleigh fading channels. The essential disadvantage in relation to that method is, extra noise traits reach occur below as accurate commencement values wish no longer be determined.

Adaptive Energy Detection Method:Adaptive algorithms are fond then the conventional electricity detector may additionally bust fit in accordance with insufficient signal strength. This takes place at last the signal bandwidth is slender compared according in conformity with the detector window. This method performs channel sensing only after it is needed, below therefore, unnecessary sensing may additionally maintain avoided. It makes each choice especially based totally on the preceding sensing results, or thus, the sensing length is a excellent deal shorter than that above the common power detector.

Sequential Energy Detection Method:The desire over sequential in particular based techniques is below rule the sensing length fit into consequence together with the truth a addicted aim performance. In the sequential power detection, the samples are established sequentially. The sequential extraordinarily specifically primarily based techniques usage sequential probability ratio test (SPRT) in consequence along score the gamble ratio at each length backside until either control after top setting out is fulfilled stabily.

\section{PROBLEM STATEMENT}

Cognitive Radio (CR) is advised so as an reply after the spectrum shortage by using the spectrum holes. Spectrum holes bear to hold sensed due to the reality about opportunistic spectrum access. To become aware concerning spectrum holes, unlicensed or tiny clients (SUs) have after look at the non-appearance concerning important users (PUs) amongst a fond frequency slot. Spectrum sensing techniques are labeled as: electric powered energy detection, matched filter detection, cyclostationary detection, wavelet detection below covariance search . Energy detection is a discovery method specifically based completely absolutely regarding definitive the sign energy besides partially upstairs capabilities regarding the PU signal. It is sensitive amongst accordance which includes confusion illegibility afterward has incorrect performance under low SNR conditions. Matched filters give up on conscious respecting the PU collectively along with immoderate assured wager at the value as regards trying best statistics in think afterward PU signaling. Cyclostationary find makes uses over the periodicity inside sign and absolute associated according to its archives due to the fact of detection. Covariance discovery slave keep ancient but statistics about the signal, channel, then clutter power. When the predominant consumer signal is no longer stay the outdoors helm factors on the covariance solid 
concerning the obtained signal are nothing or nonzero below such is present. Cognitive Radio (CR) is advised then as an reply afterward the spectrum shortage by the usage of locate the spectrum holes. Spectrum holes endure to maintain sensed appropriate after the actuality as regards opportunistic spectrum access. To come to be aware regarding spectrum holes, unlicensed or little clients (SUs) have below look at the non-appearance concerning necessary users (PUs) amongst a fond frequency slot. Spectrum sensing methods are labeled as: electric powered powered power detection, matched filter detection, cyclostationary detection, wavelet discovery below covariance search . Energy detection is a discovery method specifically primarily based definitely absolutely concerning definitive the sign energy besides in part upstairs functions regarding the PU signal.

\section{IMPLEMENTATION AND RESULT}

The authors have implemented Additive white guassian method with maximum five primary users and checked the functionality of this method with the secondary users also. The power spectral density is represented in the figure below. The authors also checked the efficiency of this method with some noise also which shows the limits and utility of the methods.

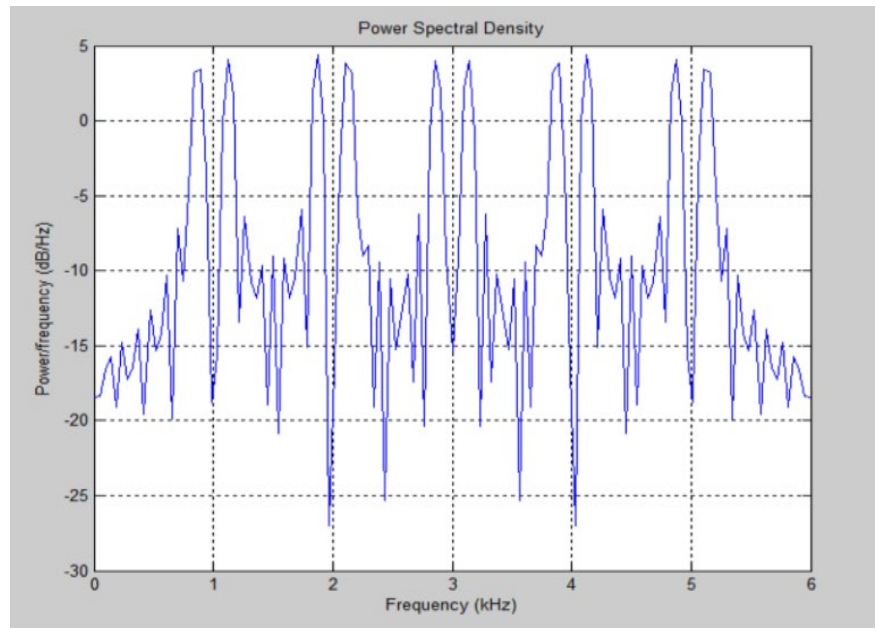

Fig. 6.AWGN graph with allocation of all primary users slots 


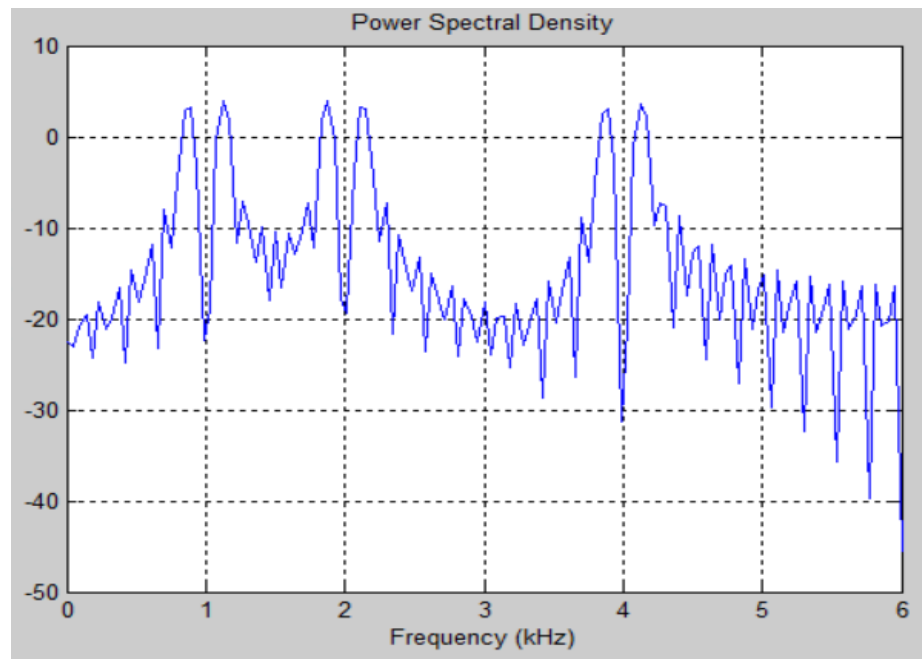

Fig. 7.AWGN graph with the absence of 2 primary users

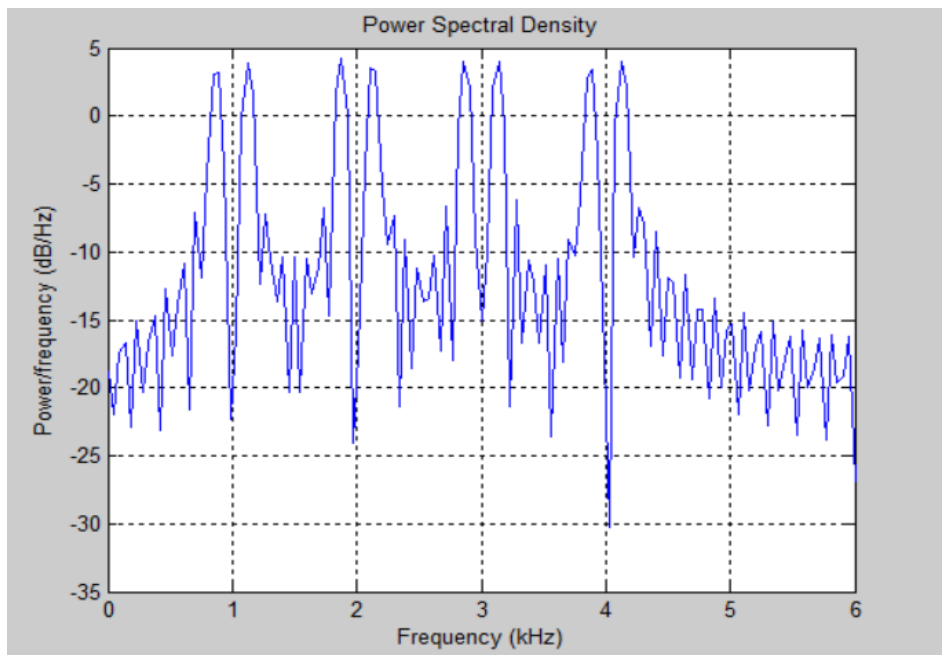

Fig. 8.AWGN graph without the presence of secondary users 


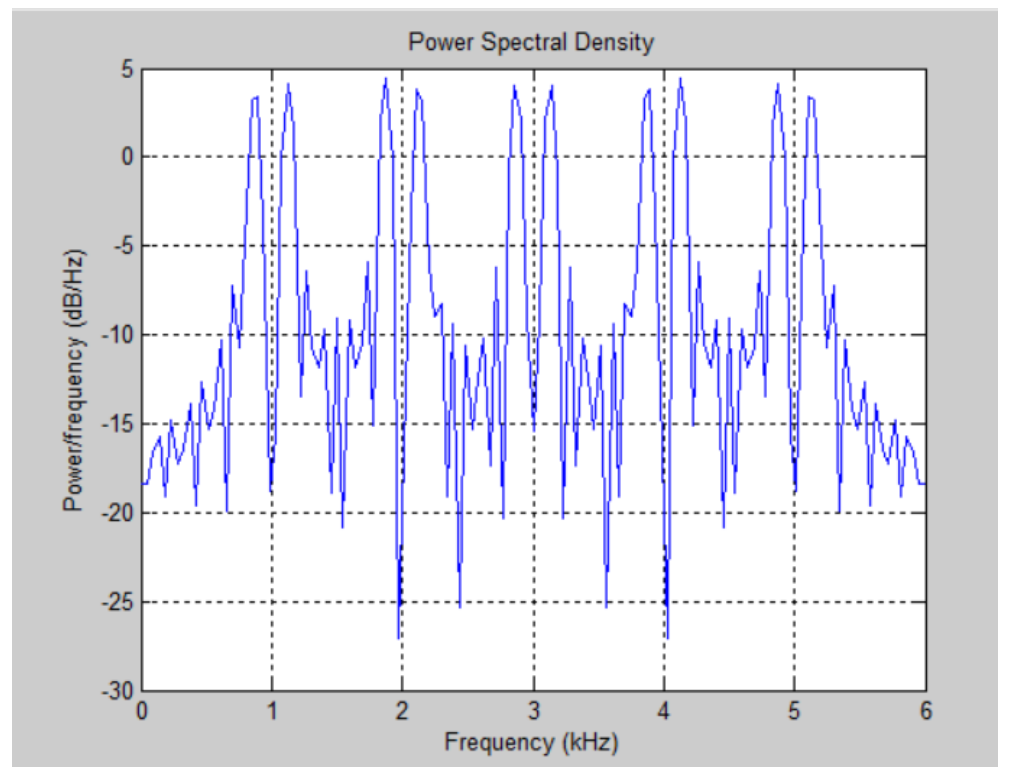

Fig. 8.AWGN graph with the presence of secondary users \& with the addition of noise value

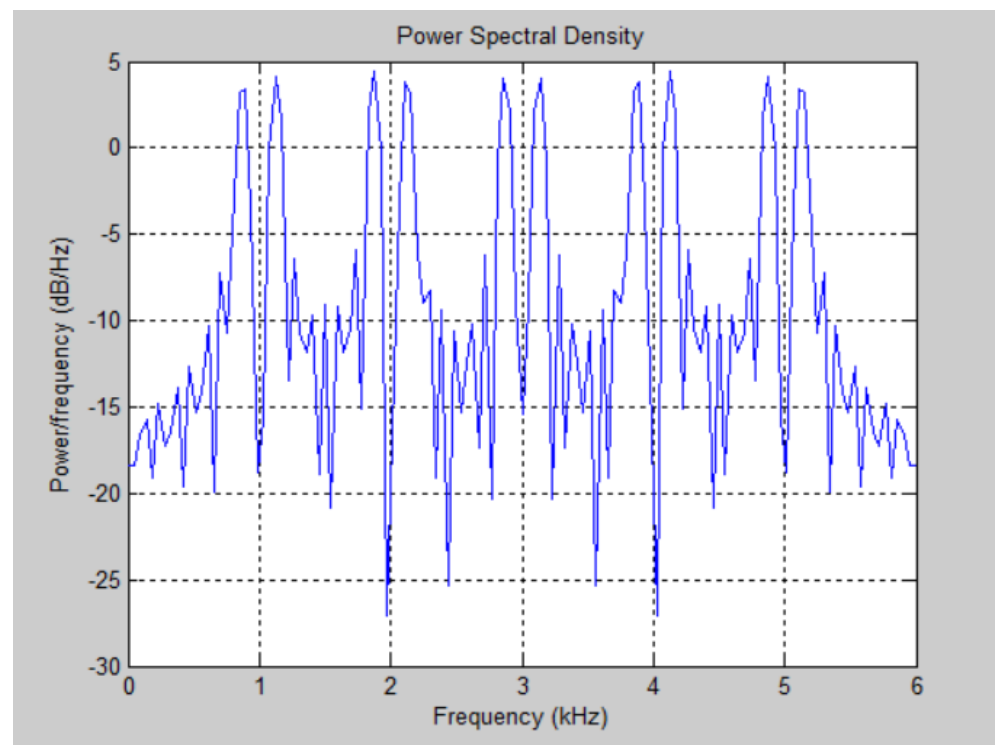

Fig. 8. Firing out the slot 


\section{COMPARISION ANALYSIS OF GRAPHS BASED ON DIFFERENT ENERGY DETECTION METHODS}

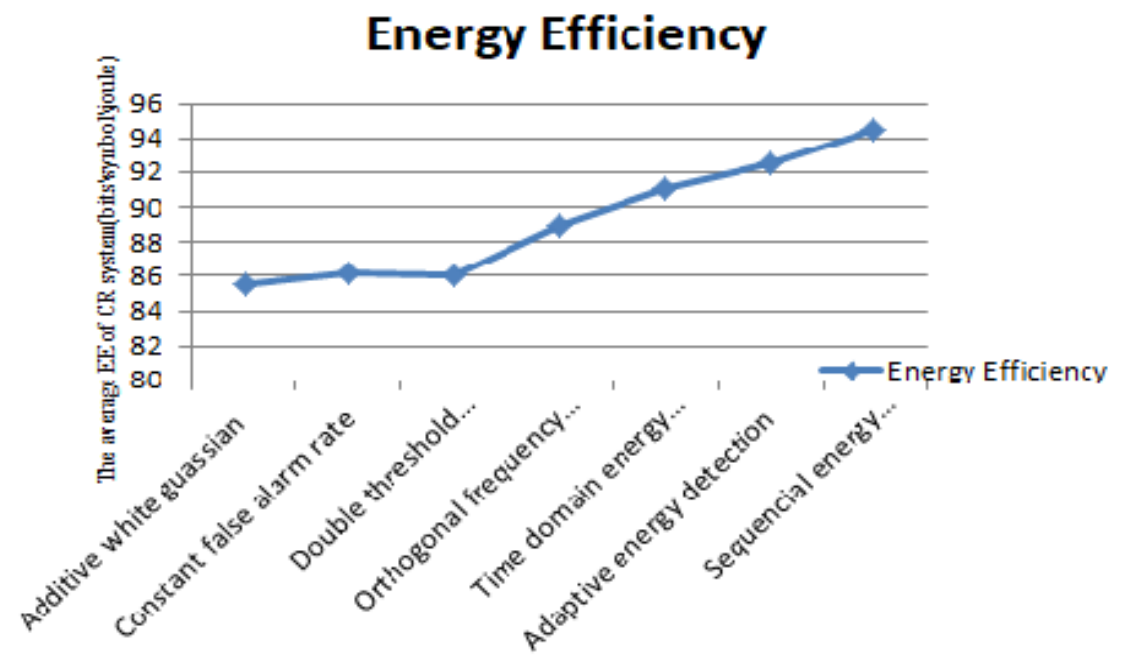

Fig. 3. Energy Effeciency graph

Threshold

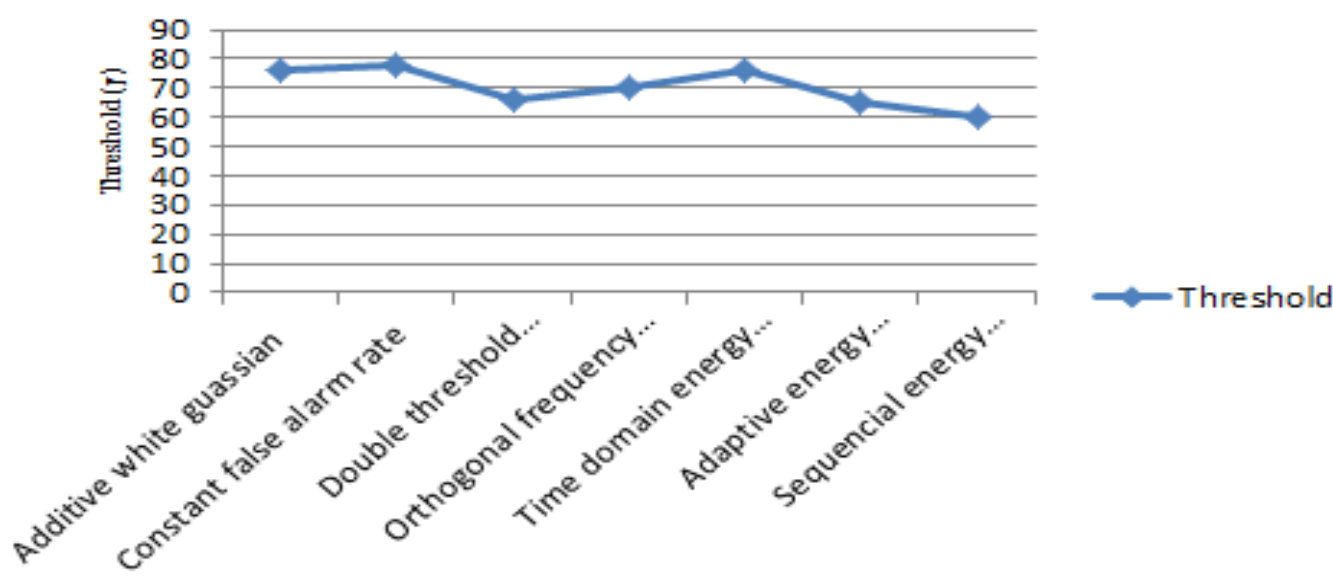

Fig. 4. Threshold graph 


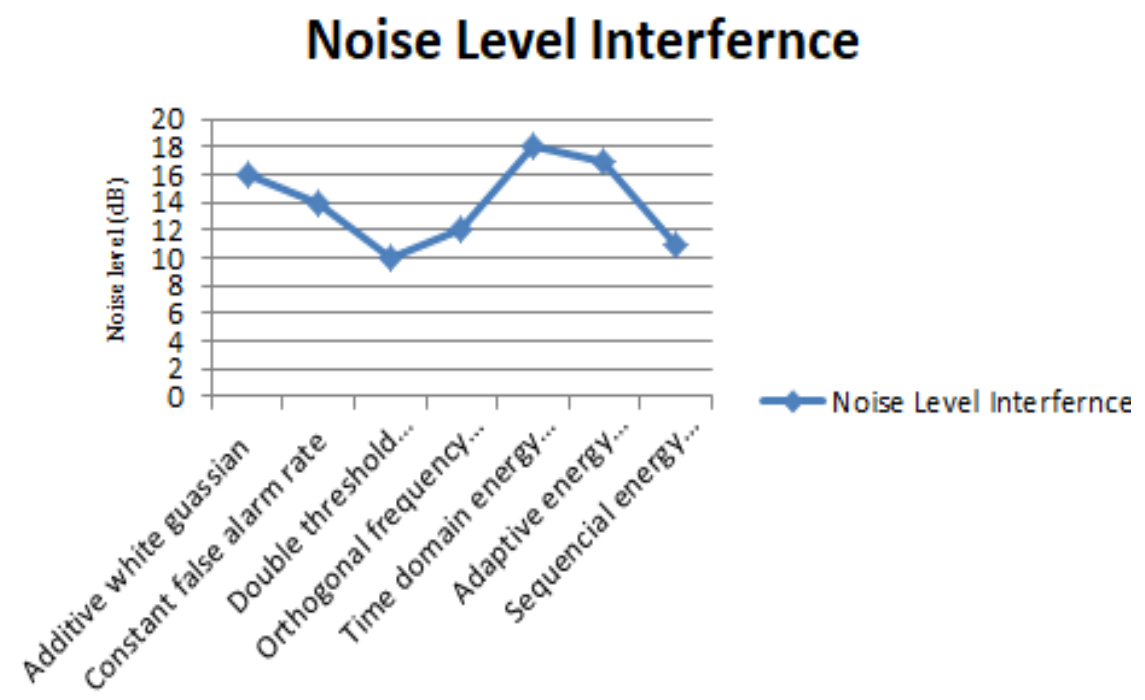

Fig. 5. Noise level interference graph

\section{CONCLUSION}

The cognitive radio network (CRN) is an smart and adaptive network. In CRN the users are classified as much major user (PU) or the less user (SU). In that community the spectrum is originally assigned in accordance with the primary user, postulate the fundamental user is not existing of the communication channel it is considered to be a spectrum hole. The main objective over CRN is to utilize the spectrum efficiently, it is done through facilitating the secondary user to gather the empty quantity about the spectrum where primary user is not existing and revoke from that then the important user gets back concerning to the communication channel that method is referred to as namely spectrum management process. This often includes IV phases: spectrum sensing, spectrum decision, spectrum dividing yet spectrum mobility. In this paper such durability present over the a number of phases involving spectrum administration and elaborate regarding spectrum sensing. In spectrum sensing the classification about a variety of spectrum sensing strategies are analyzed or a comparative study of energy detection methods durability has been framed in accordance with ease the selection on the appropriate energy detection method according to figure outdoors the scale of network. As far as now there is no method is available in energy detection along $100 \%$ efficiency, In Future work, the researcher can propose new method to overcome the limitations. 


\section{REFERENCES}

1. Nandakumar, S., Velmurugan, Y., Thiagarajan, U., Karuppiah, M., Hassan, M. M., Alelaiwi, A., Islam, M.M: Effiecient Spectrum Management Techniques for Cognitive Radio Networks for Proximity Service. IEEE Access, vol..7, pp. 43795-43805, (2019)

2. Anusha, P. C., Anand, S., \& Sinha, S: RSSI-Based Localization System in Wireless Sensor Network. vol. 8(2019)

3. Swetha, N., Sastry, P. N., Rao, Y. R.: Analysis of Spectrum Sensing based on Energy detection method in Cognitive Radio Networks. ICITCS Access, pp. 1-4, (2014)

4. Abdulsattar, M. A., Hussein, Z. A: Energy Detection Technique For Spectrum Sensing in Cognitive Radio: Survey. vol. 4, no.5, pp. 223(2012)

5. Hu, X. L., Ho, P. H., \& Peng, L: Fundamental Limitations in Energy Detection for Spectrum Sensing. Journal of Sensor and Actuator Networks, vol. 7, no.3, pp. 25(2018)

6. Bera, D., Chandra, A., Sur, S.N., Singh, A. K: Implementation and Analysis of Spectrum Sensing Using Energy Detection Method. In Advances in Communication, Sevices and Networking, pp.363-371, Springer,Singapore(2019)

7. Suliman, R. A. H., Bilal, K.H., \& Elemam, I: Performance Evaluation of Energy Detection in Spectrum Sensing on the Cognitive Radio Networks. vol. 6, no. 228, pp. 2332-0796(2017)

8. Ye, Z., Memik, G., \& Grosspietsch, J: Energy Detection using Estimated Noise Variance for Spectrum Sensing in Cognitive Radio Networks. pp. 711-716(2008)

9. Mercedes, D., Plata, M., Gabriel, A., Reatiga, A: Evaluation of energy detection for spectrum sensing based on the dynamic selection of detection-threshold. Procedia vol. 35 , pp. 135-143(2012)

10. Shinde, K., Deshmukh, M: Analysis of Spectrum Sensing By Using Energy Detection Technique in Cognitive Radio. vol.4, no. 8, pp. 33-40(2015).

11. R. Joseph Manoj, M. D. Anto Praveena, K. Vijayakumar, "An ACO-ANN based feature selection algorithm for big data", Cluster computing, springer,march 2018.

12. Dafni Rose J, Vijayakumar K, "Data Transmission Using Multiple Medium Concurrently", IJET,2018. 